\title{
Early Cell Loss Associated with Mesenchymal Stem Cell Cardiomyoplasty
}

\author{
Maria C. Collins ${ }^{1}$, Joel L. Moore, Jr. ${ }^{2}$, Brian J. Burrows ${ }^{3}$, Alan P. Kypson ${ }^{1}$ and \\ Barbara J. Muller-Borer ${ }^{*}, 1$ \\ ${ }^{I}$ Department of Cardiovascular Sciences, The Brody School of Medicine at East Carolina University, NC, USA \\ ${ }^{2}$ The University of North Carolina at Chapel Hill, Chapel Hill, NC, USA \\ ${ }^{3}$ Duke University Medical Center, Durham, NC, USA
}

\begin{abstract}
Background: Human mesenchymal stem cells (hMSCs) show potential for therapeutic cellular cardiomyoplasty. However, a range of delivery methods, including direct intramyocardial injection, have resulted in poor engraftment in vivo. We used the in vivo rat heart model to study hMSC engraftment and retention in a normal beating heart. Materials and Methods: HMSCs transfected with green fluorescent protein were injected into the left ventricle (LV) of immunocompetent rats. Hearts were cryopreserved 30 minutes (Group A), 24 hours (Group B), and 5 days (Group C) post hMSC delivery. HMSC retention was estimated using confocal fluorescence microscopy and immunohistochemistry. Measured values were compared to projected GFP-positive cellular volumes. Immunohistochemical analyses probed for the presence of human cells with human prolyl 4-hydroxylase beta $(\mathrm{p} 4 \mathrm{~h} \beta)$ and an immune response with murine monocyte/macrophage antigen (CD68). Results: HMSC retention decreased significantly from 30 minutes to 5 days $(\mathrm{p}<0.05)$. In Group A the projected GFP positive cellular volume of $31 \%$ correlated with measured values and was significantly greater than the $1 \%$ predicted cellular volume in Group C. Moreover, human $\mathrm{p} 4 \mathrm{~h} \beta$ was detected in Groups A and B, and not in Group C. Conversely, CD68 was detected in Groups B and C and not in Group A. Conclusions: In immunocompetent rats, engraftment and retention of hMSCs delivered intramyocardially significantly declines over a five day period. The influx of monocytes/macrophages suggests an unfavorable micro-environment for exogenous stem cell survival, confirmed by the absence of human cells detected five days post injection.
\end{abstract}

Keywords: Mesenchymal stem cells, cardiomyoplasty, engraftment, intramyocardial injection, cardiac.

\section{INTRODUCTION}

Recently, cellular cardiomyoplasty for myocardial repair has received significant attention as numerous cells demonstrating the potential for differentiation towards a cardiomyocyte phenotype have been identified. Embryonic stem cells, induced-pluripotent stem cells, umbilical cord blood cells, resident cardiac stem cells, adipose derived stem cells and mesenchymal stem cells (MSCs) are currently under investigation [1-5]. While various delivery methods have been used, research suggests that direct intramyocardial injection may result in higher cell retention with an increased benefit to cardiac function $[6,7]$.

Regardless of their therapeutic potential, the survival and engraftment of transplanted cells beyond a few hours is vital in their ability to provide a beneficial effect. However, studies have reported low levels of cell retention across a variety of delivery methods $[8,9]$. These reports suggest that up to $90 \%$ of transplanted cells are either lost or die soon after delivery due to hypoxia, myocardial inflammation, or the physical stresses associated with the implantation procedure [10]. Even with direct intramyocardial delivery, the majority of delivered cells may be lost due to mechanical washout, especially in a beating heart [11,12]. Transplanted

*Address correspondence to this author at the 115 Heart Drive, East Carolina Heart Institute, Room \#2247, Greenville, NC 27834, USA;

Tel: 252-744-2546; Fax: 252-744-3648; E-mail: mullerborerb@ecu.edu cells that remain in the host tissue are further challenged by biological factors such as myocardial inflammation and potential immune reactions.

As early cell loss has become apparent, in vivo studies have attempted to quantify the retention of transplanted cells in the host myocardium. Most non-invasive imaging approaches such as magnetic resonance imaging can identify very large numbers of cells [13]. For more sensitive approaches, researchers have utilized labeled cells with quantum dots or cells transfected to express a fluorescent protein [14]. Herein, we apply an established method to estimate the retention of human MSCs transfected with a gene expressing a green fluorescent protein (GFP-hMSCs) injected into normal rat myocardium [15]. Cell retention estimates correlate with measured values, confirming that significant cell loss occurs within 5 days of cardiomyoplasty. Factors such as hypoxia, washout, and the physical stresses on the cells associated with direct injection are possible causes of cell loss along with the slower biological processes.

\section{MATERIAL AND METHODS}

\section{hMSC Preparation}

GFP-hMSCs were obtained from Tulane University at passage 3 (Tulane Center for Gene Therapy NIH, Grant \# P40RR017447), cultured in Dulbecco's Modified Eagle Medium (DMEM) supplemented with $16.5 \%$ fetal bovine serum (Hyclone, Thermo Fisher Scientific, Waltham, MA), 
penicillin (100 units $/ \mathrm{ml})$, streptomycin $(100 \mu \mathrm{g} / \mathrm{ml})$ amphotericin B solution $(0.25 \mu \mathrm{g} / \mathrm{ml})$ and incubated at $37^{\circ} \mathrm{C}$ and $5 \% \mathrm{CO}_{2}$. At $80 \%$ confluency GFP-hMSCs were harvested using TrypLE Express, counted on a hemacytometer using the trypan blue method, washed twice with sterile phosphate-buffered saline (PBS, USB Corp., Cleveland, $\mathrm{OH})$, and resuspended at a concentration of $5 \times 10^{5}$ cells per $10 \mu 1$ of sterile PBS. GFP-hMSCs were maintained on wet ice with a cell viability of $96 \%$ or better observed prior to injection. (All media and supplements supplied by Invitrogen, Carlsbad, CA unless otherwise stated).

\section{Surgical Protocol}

All surgical procedures were performed in accordance with accepted guidelines for the care and treatment of experimental animals of the National Institutes of Health [16]. Appropriate Institutional Animal Care and Use Committee approval from East Carolina University was obtained. Female Sprague Dawley rats (Charles River, Wilmington, MA) weighing $300-325 \mathrm{gm}$ were anesthetized with an intraperitoneal injection of ketamine $(90 \mathrm{mg} / \mathrm{ml})$ and xylazine $(10 \mathrm{mg} / \mathrm{ml})$ at $0.1 \mathrm{ml} / 100 \mathrm{gm}$ weight, intubated, and ventilated (Harvard Apparatus, Holliston, MA). A left thoracotomy in the fourth intercostal space allowed for adequate exposure of the heart. GFP-hMSCs suspended in sterile PBS $\left(5 \times 10^{5} / 10 \mu \mathrm{l}\right)$ were injected into the anterior left ventricular (LV) wall of the beating heart using a $25 \mu 1$ syringe with a $30 \mathrm{G}$ needle (Hamilton, Reno, Nevada). After injection of GFP-hMSCs the surgical site was closed, and animals were recovered. Animals were randomly assigned to one of three survival groups: Group $A, 30$ minutes $(n=5)$; Group B, 24 hours $(\mathrm{n}=5)$; and Group C, 5 days $(\mathrm{n}=7)$. Control animals for each group were given an injection of 10 ul sterile saline.

\section{Tissue Preparation}

All animals were euthanized at the appropriate time points with $\mathrm{CO}_{2}$ exposure. Hearts were rapidly excised and rinsed in PBS. The LV was removed, embedded in optimal cutting temperature compound (Tissue-Tek Sakura, Torrance, CA), snap-frozen, and stored at $-80^{\circ} \mathrm{C}$. The LV was cut along a sagittal plane, parallel to the septum, in 10 $\mu \mathrm{m}$ sections spanning the epicardial to endocardial surface, shown in Fig. (1). Every other tissue section was analyzed by fluorescence microscopy to detect the presence of GFP positive cells. Immunohistochemistry for human cells and rodent immune cells was performed on tissue sections where GFP positive cells and/or the injection site was identified.

\section{Immunohistochemistry}

Left ventricular tissue sections were fixed in $4 \%$ paraformaldehyde in PBS, permeabilized with $0.1 \%$ Triton $\mathrm{X}-100$ and blocked with $3 \%$ bovine serum albumin (BSA, Sigma-Aldrich, St. Louis, MO). The presence of hMSCs in the LV was demonstrated using a monoclonal antibody against human prolyl 4-hydroxylase beta $(\mathrm{p} 4 \mathrm{~h} \beta$, Millipore, Billerica, MA) and Alexa Fluor 568 goat anti-mouse secondary antibody (Invitrogen, Carlsbad, CA). GFP-hMSCs grown on coverslips served as antibody control. To identify rat monocytes and macrophages, a monoclonal antibody against CD68 (Millipore, Billerica, MA) was used and rat heart tissue without GFP-hMSCs injection served as antibody control. All tissue sections were counter-stained with 4',6-diamidino-2-phenylindole, dihydrochloride (DAPI, Invitrogen, Carlsbad, CA) to identify cell nuclei.

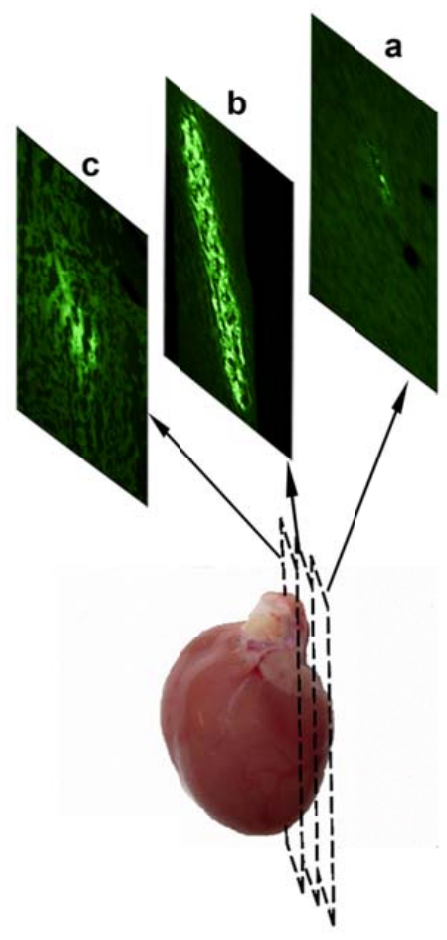

Fig. (1). Tissue sections demonstrate GFP-hMSC distribution in LV. The LV was sectioned along a sagittal plane, parallel to the septum, in $10 \mu \mathrm{m}$ sections spanning the epicardial (a) to endocardial (c) surface. Panels a- c illustrate representative sections through the LV, epicardium, mid-myocardium and endocardium, identifying GFP-hMSCs in each section.

\section{Imaging}

Tissue sections were imaged on a Zeiss Axiovert inverted fluorescence microscope equipped with an Axiocam Mrc5 camera (Carl Zeiss, Germany). Sequential fluorescence images of the sectioned LV were acquired with a $5 x$ objective, identifying the GFP positive regions. For each animal, an average of 88 tissue sections were imaged, spanning an average depth of $1.5 \mathrm{~mm}$. The GFP positive fluorescent images were converted to binary images using ImageJ software (http://rsb.info.nih.gov/ij/index.html). The binary images were used to calculate an average area of GFP positive cells. The Calvieri method [15] was used to estimate the GFP positive volume in each LV. For each image, the mean GFP positive area was calculated $\left(\mu \mathrm{m}^{2}\right)$ and summed. The GFP positive volume, estimated as $\mu \mathrm{m}^{3}$, was calculated with equation 1 where the interval between tissue sections was $20 \mu \mathrm{M}$.

$\sum$ (Area of GFP positive cells)(Interval between tissue sections $)=$ GFP positive volume

To estimate the average cell volume of the hMSC injection, confocal fluorescent images of hMSCs in suspension 
were acquired. The average measured diameter of the spherical cells was $10 \mu \mathrm{m}$, corresponding to an average cellular volume of $524 \mu \mathrm{m}^{3}$ per cell. This cell volume approximation was used to estimate the percentage of hMSCs delivered to and retained in the LV where each injection consisted of $5 \mathrm{x}$ $10^{5}$ cells $/ 10 \mu \mathrm{PBS}$. Equation 2 was used to estimate the hMSC injection and retention volume in GFP positive tissue sections.

Estimated \# of GFP-hMSCs = GFP positive volume $\left(\mu \mathrm{m}^{3}\right) /$ Average cell volume $\left(\mu \mathrm{m}^{3}\right)$

Immunofluorescent images of human $\mathrm{p} 4 \mathrm{~h} \beta$ and rodent CD68 were acquired with a Zeiss Axiovert fluorescence microscope using a 10x objective.

\section{Statistics}

The data is presented as mean \pm SEM. The statistical significance was determined using an analysis of variance (ANOVA) and Tukey post hoc analysis where appropriate. A value of $p<0.05$ was considered statistically significant.

\section{RESULTS}

\section{Animal Survival}

A total of 25 animals received LV injections of GFPhMSCs. Seventeen animals survived and were analyzed for GFP-hMSC retention (4 were lost due to early death, and 4 due to technical errors).

\section{Distribution of GFP-hMSC in LV}

The estimated number of GFP-hMSCs retained at each injection site was determined from measurements acquired from the serial tissue sections as described in the Methods and shown in Fig. (1). The distribution of GFP-hMSCs in the LV was variable despite a consistent delivery technique. The variability of five GFP-hMSC injections measured in the LV at 30 minutes after delivery (Group A) is shown in Fig. (2). The average GFP positive areas ranged from 12,302 \pm $13,126 \mu \mathrm{m}^{2}$ to $93,719 \pm 84,197 \mu \mathrm{m}^{2}$ with GFP-hMSC distribution in animals A-1, A-2 and A-3 distributed throughout the LV. However, GFP-hMSC delivery in animal A-4 was deeper in the LV and GFP-hMSC delivery in animal A-5

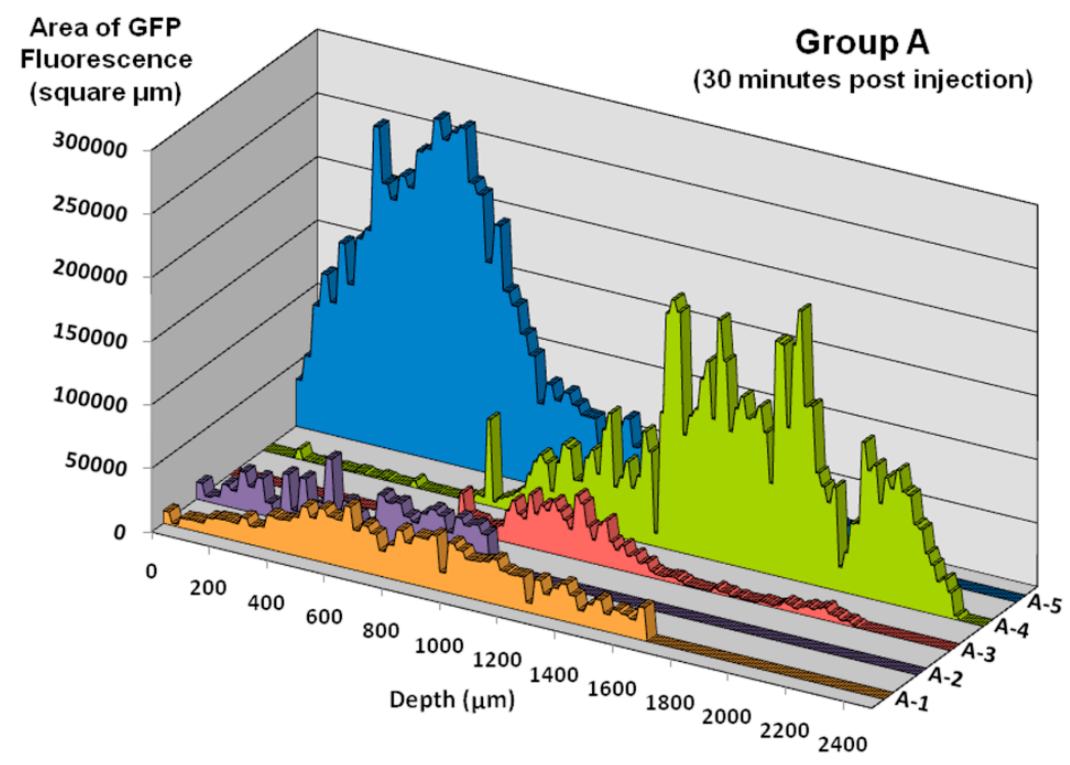

Fig. (2). Variability of hMSC injections measured 30 minutes after delivery (Group A). Graph shows GFP-hMSC distribution in the LV from five hMSC injections (A-1 through A-5). The average GFP positive area ranged from $12,302 \pm 13,126 \mu \mathrm{m}^{2}$ to $93,719 \pm 84,197 \mu \mathrm{m}^{2}$. The GFP-hMSCs in A-1, A-2 and A-3 distributed throughout the LV, while the GFP-hMSCs of A-4 were delivered deeper in the LV and the GFP-hMSCs of A-5 were closer to the epicardium.

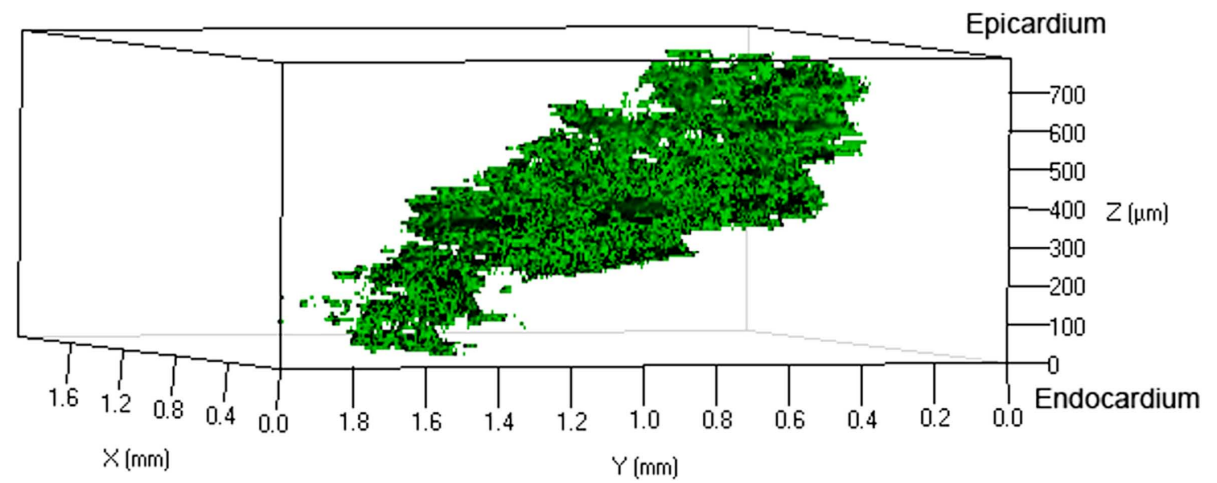

Fig. (3). Three-dimensional reconstruction of GFP positive volume from Group B LV sections. The Z axis represents the epicardial to endocardial surface. Note the distribution of the GFP-hMSCs throughout the LV. 
was closer to the epicardium. A representative image rendered from the $10 \mu \mathrm{m}$ fluorescent tissue sections illustrates a three-dimensional GFP-hMSC volume distribution at 24 hours (Fig. 3)

\section{GFP-hMSC Retention}

The average GFP positive volume calculated from the LV sections in Group A (30 minutes), Group B (24 hours), and Group C (5 days) are shown in Fig. (4). A significantly greater GFP positive volume was detected in Group A vs. Group C $\left(8.2 \times 10^{7} \pm 3.4-\times 10^{7} \mu \mathrm{m}^{3}\right.$ vs. $1.6 \times 10^{6} \pm 1.0 \times 10^{6}$ $\left.\mu \mathrm{m}^{3}, \mathrm{p}<0.05\right)$. These volumes correspond to an estimated cell retention of $31 \%$ or $1.6 \times 10^{5} \pm 0.1 \times 10^{5}$ cells for Group A,
$9 \%$ or $4.4 \times 10^{4} \pm 0.5 \times 10^{4}$ cells for Group B and $1 \%$ or 5.2 $\times 10^{3} \pm 5.2 \times 10^{3}$ cells for Group C. The presence of human cells was evaluated with the co-localization of human $\mathrm{p} 4 \mathrm{~h} \beta$ with GFP-hMSCs in the LV. Only LV tissue sections from Groups A and B stained positive for human $\mathrm{p} 4 \mathrm{~h} \beta$ which colocalized with the GFP-hMSCs. Representative images are shown in Fig. (5). In LV sections from Group C human $\mathrm{p} 4 \mathrm{~h} \beta$ was not detected although negligible amounts of GFP fluorescence was detected.

\section{Immune Response}

To ascertain the role of immune cells on hMSC engraftment and retention LV tissue sections were probed for the

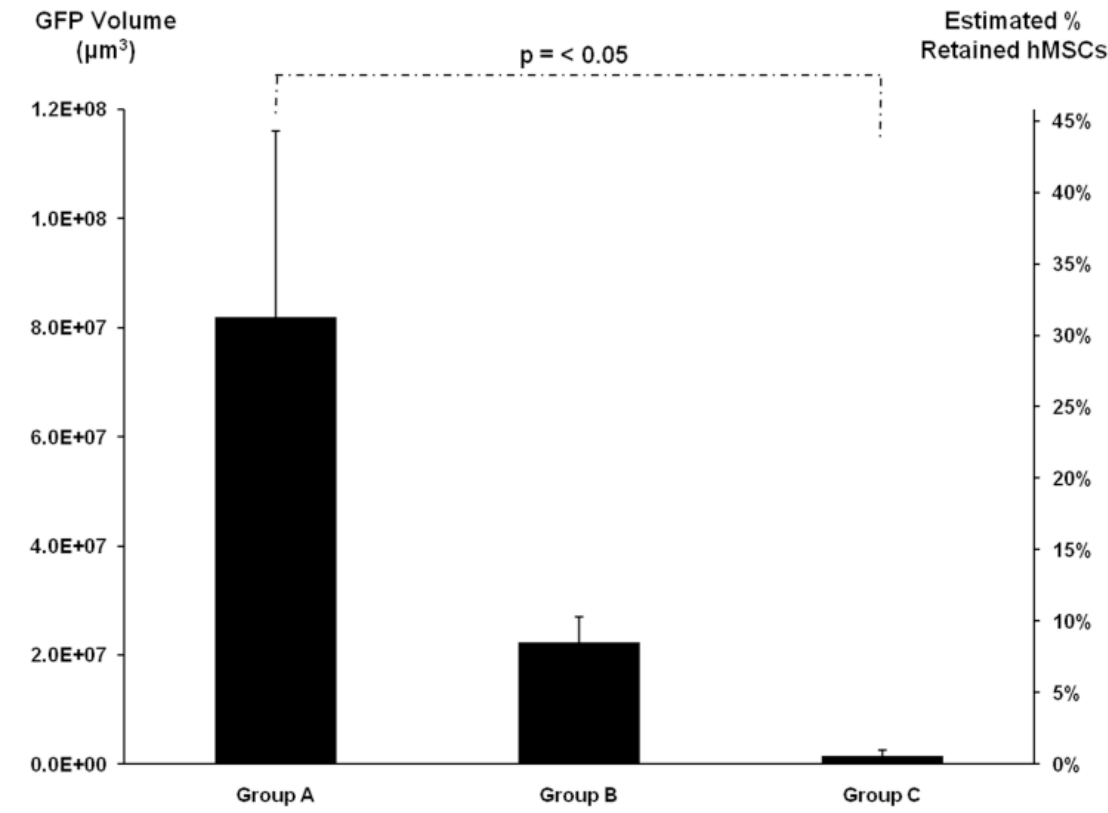

Fig. (4). Average GFP positive volume $\left(\mu \mathrm{m}^{3}\right)$ measured from imaged tissue sections (mean \pm SEM ) corresponds to estimated percentage of hMSCs retained in cardiac tissue after transplantation. Thirty minutes post-injection, $31 \%$ of the GFP-hMSCs were detected in the LV. This contrasts with the $1 \%$ of GFP-hMSCs detected at 5 days post injection. A significant decrease in GFP-hMSC was detected in Group A vs. Group C $(\mathrm{p}<0.05)$.

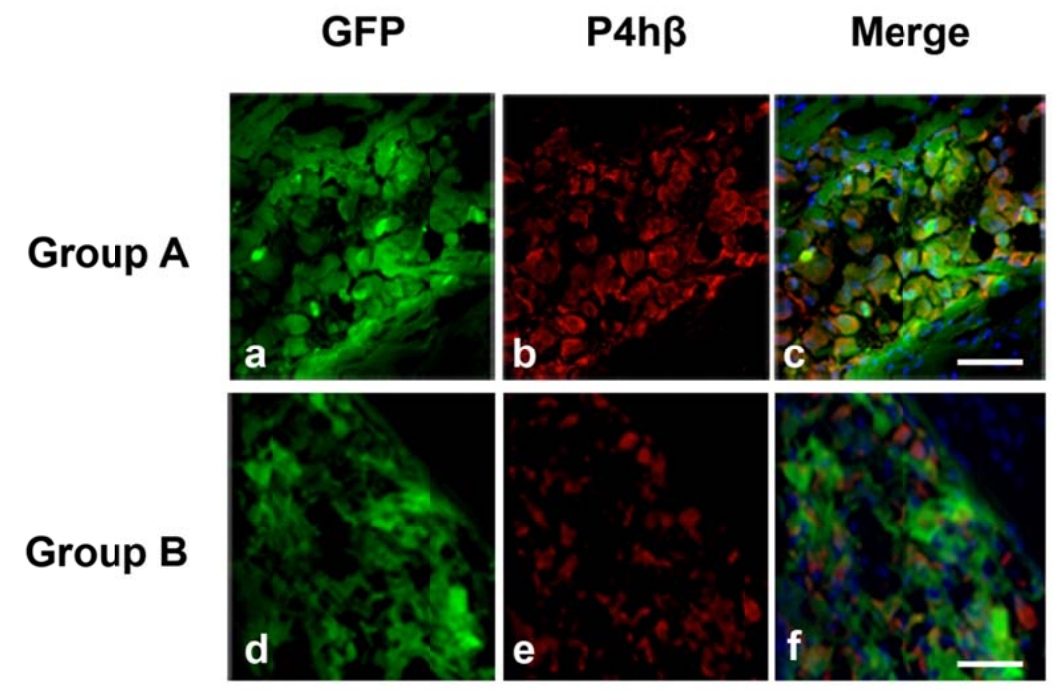

Fig. (5). Fluorescence images identifying hMSCs in rat LV tissue sections 30 minutes (Group A) and 24 hours (Group B) after hMSC transplantation. Panels (a) and (d) show GFP-hMSCs (green), panels (b) and (e) show human p4hß (red) with the merged image in panels (c) and (f) demonstrating co-localization of the GFP with the human antibody. Cell nuclei are identified with DAPI (blue). Scale bar $=200 \mu \mathrm{m}$. 
presence of monocytes/macrophages using a rat CD68 antibody. In Group $\mathrm{C}$, rat monocytes/macrophages were detected at the site of injection and correlated with decreased retention of GFP-hMSCs over the 5 day interval. Moreover, rat monocytes/macrophages were detected at the injection site in Group C Control LV (saline only). Representative images are shown in Fig. (6). Minimal CD68 was detected in the LV of Group B and no CD68 was detected in Group A. Rat heart tissue without GFP-hMSCs injection served as antibody control.

\section{DISCUSSION}

Delivery methods for cellular cardiomyoplasty vary greatly. Common techniques include intracoronary injection via catheterization [17-19], direct intramyocardial injection [20] or attachment of a pre-seeded bioscaffold to the heart [21]. Intramyocardial injections, although invasive, offer the most direct and concentrated delivery of cells to the cardiac region of interest. Several reports have shown short-term benefits of MSC injections administered in this manner [20, $22,23]$. These transitory benefits, such as an increased ejection fraction in infarcted hearts, may be due to delivering cells that could potentially integrate into the host myocardium, secrete soluble growth and angiogenic factors, or recruit the host's own resident stem cells [1, 24, 25]. Although the exact mechanisms remain unknown, in order for cellular cardiomyoplasty to provide any long-term functional benefits, delivered cells should ideally survive and be retained within the myocardium beyond a few days. As shown in this study, we demonstrate that only a small percentage of delivered hMSCs are found very early post delivery (30 minutes) to the myocardium despite being directly injected. In fact, the number of retained cells significantly declines over the ensuing five days. The concomitant loss of human $\mathrm{p} 4 \mathrm{~h} \beta$ expression confirmed this finding. Furthermore, we have shown that within 5 days after intramyocardial injection, there exists a significant immune response creating a hostile environment for hMSCs.

Cell loss during intramyocardial injection can occur for a variety of reasons. The beating heart is the most mechaniccally active organ in the body. In a rat model, average heart rates can exceed 240 beats per minute. Obvious technical challenges associated with injecting into the beating myocardium exist. Shallow injections may result in excessive washout whereas deeper injections may inadvertently deliver hMSCs into the left ventricle and circulatory system. Here, we have shown that approximately $31 \%$ of injected hMSCs were retained at the injection site in a beating rat heart model at 30 minutes after delivery. Teng and colleagues [12] documented massive loss (ranging from 90-98\%) of microspheres (surrogates for cells) within 10 minutes after injection in beating normal rat model even with a pursestring occlusion at the injection site. In a porcine model, our group has shown that approximately $10 \%$ of injected microspheres were retained within the injection site. Interestingly, we also demonstrated that even with cardiac standstill using cardiopulmonary bypass, retention rates of microspheres were not significantly different [11]. This would suggest that loss of injected material was due to other factors besides mechanical loss at the injection site due to the contractile force of the myocardium. In fact, it would imply that there was an internal washout within the myocardial veins and

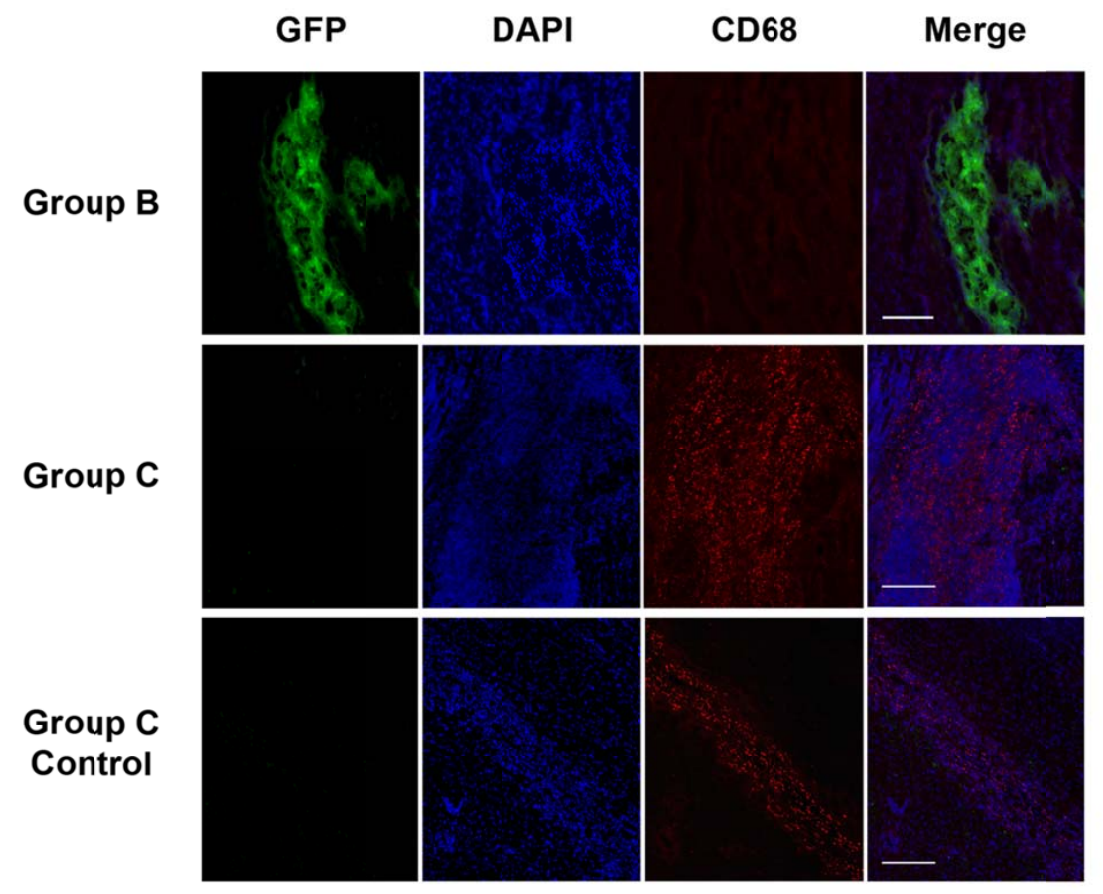

Fig. (6). Immune response at 24 hrs and 5 days post hMSC transplantation. Fluorescent images of representative LV sections 24 hours (Group B), 5 days post hMSC injection (Group C) and 5 days post saline injection (Group C control). GFP-hMSCs (green), cell nuclei with DAPI (blue), immune response by the macrophage marker CD68 (red) and merged images are shown (overlay). Note the decrease in GFP fluorescence and increase in CD68 in the LV at 24 hours and 5 days post GFP-hMSCs injection versus control. At 24 hours post saline injection Group B control showed no difference in CD68 fluorescence (image not shown). Scale bar $=200 \mu \mathrm{m}$. 
lymphatics taking away the majority of injected material. When examined, microspheres were found in organs besides the heart. Presumably, similar factors are at work in our current model described here, resulting in an acute loss of delivered hMSCs from the host myocardium. Others have documented similar results [26]. Hou and coworkers evaluated the short term fate (one hour) of radiolabeled cells delivered intramyocardially in a beating ischemic heart swine model [27]. They documented a retention rate ranging from $8-14 \%$ within the heart and widespread distribution within the visceral organs. Furthermore, they also showed that an average of $12 \%$ of labeled cells were retained in the delivery device after injection. For this reason, in this study, we used a Hamilton syringe which by design has no dead space.

Besides the mechanical forces associated with acute hMSC loss during intramyocardial injection, other biological influences may contribute to the poor retention rate of hMSCs within the myocardium. Recently, attention has been drawn to the hypoxic environment that might be created by the injection itself $[28,29]$. A significant amount of hMSCs are delivered to a small area and that volume significantly decreases local perfusion as evidenced by the blanching of myocardial tissue witnessed with each injection. Even within non-ischemic hearts, this may create conditions that are unfavorable for acute cell survival resulting in death of the injected hMSCs. Logically, one could then assume that smaller injectate volumes delivered to multiple areas might yield a higher cumulative retention and survival rate than one large injectate volume. Ott and colleagues have described this observation in a rat infarct model [30]. Intramyocardial cell delivery performed with smaller, more numerous, injections resulted in improved cardiac function and a larger number of engrafted cells. The authors speculated that these findings may have resulted from an increased surface to volume ratio in each injection allowing increased contact area between injected cells and host myocardium. Further investigations to confirm these observations seem warranted in light of poor retention rates observed in our study.

To locate the transplanted GFP-hMSCs we sectioned the $\mathrm{LV}$ in $10 \mu \mathrm{m}$ sections along the sagittal plane from the epicardial surface towards the endocardium. This allowed us to image the tissue at 20 um intervals, carefully documenting the hMSC distribution in each LV. This analysis clearly demonstrated the variability in delivered hMSC distribution among animals and the importance of careful sampling. Unlike many histological studies that report hMSC retention from random tissue sections, we analyzed hMSC distribution in the entire LV. The Calvieri method [15, 31-33] provided a quantitative measure of the irregular GFP-hMSC volume distribution in the LV. Using this method to estimate GFP positive volume in the LV and correlating this volume to the percent retained GFP-hMSCs revealed that over $50 \%$ of the hMSCs were lost within the first 30 minutes after delivery. These estimates agree with similar measurements of stem cell retention using positron emission tomography to locate cardiac-derived stem cells in beating rat heart infarct model [34].

Aside from the above mentioned influences that contribute to acute cell loss, there are other biological factors that contribute to chronic cell loss. Our data demonstrate that there is a continual decrease in the retention of injected
hMSCs and by 5 days injected hMSCs are virtually impossible to identify using GFP. The lack of staining for human $\mathrm{p} 4 \mathrm{~h} \beta$ in the myocardial tissue confirmed this observation. Concurrent loss of GFP and $\mathrm{p} 4 \mathrm{~h} \beta$ at 5 days suggests the transplanted hMSCs are not present, having either died or migrated elsewhere, they haven't simply just lost their ability to fluoresce. Mechanical loss and cell death can certainly continue to contribute to the significant decline during this time. However, other more chronic processes such as the host's own immune system must play a significant role in hMSC loss as well. Therefore, using a rat CD68 antibody to identify monocytes and macrophages, we were able to demonstrate that there is a considerable increase in monocytes and macrophages at the injection site. Presumably, the remaining hMSCs are further challenged by a localized cellular inflammatory response within the injection area. In contrast, there was very little evidence of an immune response 30 minutes after intramyocardial injection as demonstrated by the lack of staining for rat CD68 in Group A. Even more interesting, was the fact that we observed a significant and similar immune response at 5 days within the control group (saline injection only). This would suggest that the injection itself creates an injury within the myocardium that causes a host immune response. This finding highlights a potentially significant limitation of the intramyocardial injection as a delivery method for cell cardiomyoplasty. Further substantiating the host cellular response to the injection is our DAPI staining (nuclear counterstain) at 5 days post injection that showed a large number of cells within the injection site which were not present at 30 minutes or 24 hours after injection. Ultimately, the micro-environment that may be created by myocardial injections may not be suitable for continued survival of transplanted cells.

The use of a xenograft model of human MSCs delivered into rat myocardium has several limitations. We chose this model to maximize our ability to differentiate between host and transplanted cells using species specific markers. While we used GFP to label cells, other methods such as antibodies to human mitochondria could have been used as well [35]. Concern may be raised about the xenogenic immune response that could be elicited from these injections. However, hMSCs lack MHC Class II antigens and co-stimulatory molecules and have previously been shown to not induce xenoreactivity in immunocompetent Sprague-Dawley rats [36], although significant controversy still exits. Nevertheless, hMSCs may evade immunosurveillance [37]. For example, allogeneic MSCs injected into infarcted myocardium in a pig model showed no signs of rejection and reduced infarct size via myocardium regeneration [24]. Therefore, we elected not to immunosuppress recipient animals. However, future studies with athymic rats could further help distinguish immunological vs. non-immunological mechanisms of cell loss. Furthermore, we did not examine peripheral organs for washout of injected cells. Based on prior results, we would anticipate substantial numbers of hMSCs in the lungs, liver and spleen [11].

\section{CONCLUSION}

We show that there is significant loss of hMSCs after intramyocardial delivery that begins almost immediately after the injection is complete. This loss continues for up to 5 
days at which point almost all transplanted hMSCs have either been washed out of the myocardium or died as a result of a significant host cellular immune response. Future experiments might try to alter the delivery method. Smaller, more numerous injections may yield a higher total cell retention count than a single large injection, especially in a small heart such as the rat. Modulating the immune response locally might also improve chances of long term engraftment. Nevertheless, our data supports concerns that the optimal delivery method has not been established. Continued work in this area is imperative if cellular cardiomyoplasty is to have any meaningful long-term clinical impact.

\section{CONFLICT OF INTEREST}

None declared.

\section{ACKNOWLEDGEMENTS}

Funding provided by the Murray and Sydell Rosenberg Foundation.

\section{REFERENCES}

[1] Beltrami AP, Urbanek K, Kajstura J, et al. Evidence that human cardiac myocytes divide after myocardial infarction. New Engl J Med 2001; 344: 1750-57.

[2] Mauritz C, Schwanke K, Reppel M, et al.Generation of functional murine cardiac myocytes from induced pluripotent stem cells, Circulation 2008; 118: 507-17.

[3] Mummery C, Ward D, van den Brink CE, et al. Cardiomyocyte differentiation of mouse and human embryonic stem cells. J Anat 2002; 200: 233-42.

[4] Planat-Benard V, Menard C, Andre M, et al. Spontaneous cardiomyocyte differentiation from adipose tissue stroma cells. Circ Res 2004; 94: 223-9.

[5] Toma C, Pittenger MF, Cahill KS, Byrne BJ, Kessler PD. Human mesenchymal stem cells differentiate to a cardiomyocyte phenotype in adult murine heart. Circulation 2002; 105: 93-8.

[6] Psaltis PJ, Zannettino ACW, Worthley AG, Gronthos S. Concise Review: Mesenchymal stromal cells: potential for cardiovascular repair. Stem Cells 2008; 26: 2201-10.

[7] Perin EC, Silva GV, Assad JA, et al. Comparison of intracoronary and transendocardial delivery of allogeneic mesenchymal cells in a canine model of acute myocardial infarction. J Mol Cell Cardiol 2008; 44: 486-95.

[8] Fukuda K. Development of regenerative cardiomyocytes from mesenchymal stem cells for cardiovascular tissue engineering. Artificial Organs 2001; 25:187-93.

[9] Wang JS, Shum-Tim D, Galipeau J, Chedrawy E, Eliopoulos N, Chiu RC. Marrow stromal cells for cellular cardiomyoplasty: feasibility and potential clinical advantages. J Thorac Cardiovasc Surg 2000; 120: 999-1005.

[10] Rosenstrauch D, Poglajen G, Zidar N, Gregoric ID. Stem cell therapy for ischemic heart failure. Tex Heart Inst J 2005; 32: 33947.

[11] Hudson W, Collins MC, Dorian deFreitas D, Sun YS, Muller-Borer BJ, Kypson AP. Beating and arrested intramyocardial injections are associated with significant mechanical loss: implications for cardiac cell transplantation. J Surg Res 2007; 142: 263-7.

[12] Teng CJ, Luo J, Chiu RCJ, Shum-Tim D. Massive mechanical loss of microspheres with direct intramyocardial injection in the beating heart: Implications for cellular cardiomyoplasty. J Thorac Cardiovas Surg 2006; 132: 628-32.

[13] Kraitchman DL, Tatsumi M, Gilson WD, et al. Dynamic imaging of allogeneic mesenchymal stem cells trafficking to myocardial infarction. Circulation 2005; 112: 1451-61.

[14] Muller-Borer BJ, Collins MC, Gunst PR, Cascio WE, Kypson AP. Quantum dot labeling of mesenchymal stem cells. J Nanobiotech 2007; 5: 1-9.
[15] Gundersen HJG, Bendtsen TF, Korbo L, et al. Some new, simple and efficient stereological methods and their use in pathological research and diagnosis. APMIS 1988; 96: 376-94.

[16] National Research Council of the National Academies: Guide for the Care and Use of Laboratory Animals, NIH Publication No. 8621. Washington DC: National Academy Press 1996.

[17] Tendera M, Wojakowski W, Ruz W, et al. Intracoronary infusion of bone marrow-derived selected CD341CXCR41 cells and nonselected mononuclear cells in patients with acute STEMI and reduced left ventricular ejection fraction: results of randomized, multicentre Myocardial Regeneration by Intracoronary Infusion of Selected Population of Stem Cells in Acute Myocardial Infarction (REGENT) Trial. Eur Heart J 2009; 30: 1313-21.

[18] Dill $\mathrm{T}$, Volker Schachinger $\mathrm{V}$, Rolf $\mathrm{A}$, et al. Intracoronary administration of bone marrow-derived progenitor cells improves left ventricular function in patients at risk for adverse remodeling after acute ST-segment elevation myocardial infarction: Results of the Reinfusion of Enriched Progenitor cells And Infarct Remodeling in Acute Myocardial Infarction study (REPAIR-AMI) cardiac Magnetic Resonance Imaging substudy. Am Heart J 2009; 157: 541-7.

[19] Meyer GP, Wollert KC, Lotz J, et al. Intracoronary bone marrow cell transfer after myocardial infarction: eighteen months' followup data from the randomized, controlled BOOST (Bone marrow transfer to enhance ST-elevation infarct regeneration) trial. Circulation 2006; 113: 1272-4.

[20] Hare JM, Chaparro SV. Cardiac regeneration and stem cell therapy. Curr Opin Organ Transplant 2008; 13: 536-42.

[21] Jawad H, Lyon AR, Harding SE, Ali NN, Boccaccini AR. Myocardial tissue engineering. Br Med Bull 2008; 87: 31-47.

[22] Kim B-O, Tian H, Prasongsukarn K, et al. Cell transplantation improves ventricular function after a myocardial infarction a preclinical study of human unrestricted somatic stem cells in a porcine model. Circulation 2005; 112(suppl I): I96-I104.

[23] Schuleri KH, Feigenbaum GS, Marco Centola M, et al. Autologous mesenchymal stem cells produce reverse remodelling in chronic ischaemic cardiomyopathy. Eur Heart J 2009; 30: 2722-32.

[24] Amado LC, Saliaris AP, Schuleri KH, et al. Cardiac repair with intramyocardial injection of allogeneic mesenchymal stem cells after myocardial infarction. Proc Nat Acad Sci USA 2005; 102: 11474-9.

[25] Mangi AA, Noiseux N, Kong D, et al. Mesenchymal stem cells modified with Akt prevent remodeling and restore performance of infarcted hearts. Nat Med 2003; 9: 1195-201.

[26] Dow J, Simkhovich BZ, Kedes L, Kloner RA. Washout of transplanted cells from the heart: a potential new hurdle for cell transplantation therapy. Cardiovasc Res 2005; 67: 301-7.

[27] Hou D, Youssef E, Brinton T, et al. Radiolabeled cell distribution after intramyocardial, intracoronary, and interstitial retrograde coronary venous delivery. Circulation 2005; 112: I-150-I-156.

[28] Zhu W, Chen J, Cong X, Hu S, Chen X. Hypoxia and serum deprivation-induced apoptosis in mesenchymal stem cells. Stem Cells 2006; 24: 416-25.

[29] Potier E, Ferreira E, Meunier A, Sedel L, Logeart-Avramoglou D, Petite H. Prolonged hypoxia concomitant with serum deprivation induces massive human mesenchymal stem cell death. Tissue Eng 2007; 13: 1325-31.

[30] Ott HC, Kroess R, Bonaros N, et al. Intramyocardial microdepot injection increases the efficacy of skeletal myoblast transplantation. Eur J Cardiothorac Surg 2005; 27: 1017-21.

[31] Gundersen HJG, Jensen EBV, Kieu K, Nielsen J. The efficiency of systematic sampling in stereology - reconsidered. J Microsc 1999; 193: 199-211.

[32] Kubínová L, Janácek J. Confocal microscopy and stereology: estimating volume, number, surface area and length by virtual test probes applied to three-dimensional images. Microsc Res Tech 2001; 53: 425-35.

[33] Kubínová L, Janácek J, Ribaric S, Cebasek V, Erzen I. Threedimensional study of the capillary supply of skeletal muscle fibres using confocal microscopy. J Muscle Res Cell Motil 2001; 22: 21727.

[34] Terrovitis J, Lautamäki R, Bonios $\mathrm{M}$, et al. Noninvasive quantification and optimization of acute cell retention by in vivo positron emission tomography after intramyocardial cardiacderived stem cell delivery. J Am Coll Cardiol 2009; 54: 1619-26. 
[35] Muller-Borer BJ, Cascio WE, Esch GL, et al. Mechanisms controlling the acquisition of a cardiac phenotype by liver stem cells. Proc Natl Acad Sci USA 2007; 104: 3877-82.

[36] Grinnemo KH, Mansson A, Dellgren G, et al. Xenoreactivity and engraftment of human mesenchymal stem cells transplanted into infarcted rat myocardium. J Thorac Cardiovasc Surg 2004; 127: 1293-300.

[37] Ryan JM, Barry FP, Murphy JM, Mahon BP. Mesenchymal stem cells avoid allogeneic rejection. J Inflamm 2005; 2: 2-8.

(C) Collins et al.; Licensee Bentham Open.

This is an open access article licensed under the terms of the Creative Commons Attribution Non-Commercial License (http://creativecommons.org/licenses/bync/3.0/), which permits unrestricted, non-commercial use, distribution and reproduction in any medium, provided the work is properly cited. 